\title{
ON APPROXIMATION OF THE SOLUTIONS OF DELAY DIFFERENTIAL EQUATIONS BY USING PIECEWISE CONSTANT ARGUMENTS
}

\author{
ISTVAN GYÖRI \\ Department of Mathematics, The University of Rhode Island \\ Kingston, Rhode Island 02881 USA \\ and \\ Computing Centre of A. Szent-Györgyi Medical University \\ H - 6720 Szeged, Pecsi u. 4/a., Hungary.
}

(Received June 12, 1989 and in revised form June 30, 1989)

\begin{abstract}
By using the Gronwall - Bellman inequality we prove some limit relations between the solutions of delay differential equations with continuous arguments and the solutions of some related delay differential equations with piecewise constant arguments(EPCA). EPCA are strongly related to some discrete difference equations arising in numerical analysis, therefore the results can be used to compute numerical solutions of delay differential equations. We also consider the delay differential equations of neutral type by applying a generalization of the Gronwall - Bellman inequality.
\end{abstract}

KEY WORDS AND PHRASES. Differential equations of retarded and neutral types, differential equations with piecewise constant arguments, numerical approximation of solutions, difference equations.

1980 AMS SUBJECT CLASSIFICATION CODE. 34K, 39A, 65L.

1. INTRODUCTION.

Recently, Cooke and Wiener [4] introduced a new class of delay differential equations. These, so called delay differential equations with piecewise constant arguments (EPCA) consolidate several properties of the continuous dynamical systems generated by delay differential equations ([9]) and of the discrete dynamical systems generated by difference equations ([10]). In fact, a wide class of delay differential equations with piecewise constant arguments can be rearranged to some difference equations (see, e.g. [1], [2], [8]) which remind us of the numerical approximating equations of some delay differential equations.

Our aim in this paper is to establish some approximating results for the solutions of delay differential equations via the solutions of some related delay differential equations with piecewise constant arguments.

Consider the delay differential equation 


$$
\dot{x}(t)+p_{0}(t) x(t)+\sum_{i=1}^{m} p_{i}(t) x\left(t-\tau_{i}\right)=0, \quad t \geqslant 0
$$

where for $i=1, \ldots, m, \tau_{i}$ are positive real numbers and for $i-0,1, \ldots, m$, $P_{i}: R_{+} \rightarrow R$ are continuous functions. Set $\tau-\max _{1 \leqslant 1 \leqslant m} \tau_{i}$.

Let $[\cdot]$ denote the greatest integer function, $N$ the set of nonnegative integers. For a fixed $k \in N,(k \geqslant 1)$, set $h-\frac{r}{k}$ and define three delay differential equations with plecewise constant arguments as follows :

$$
\dot{u}(t)+p_{0}(t) u(t)+\sum_{i=1}^{m} p_{i}(t) u\left(\left[\frac{t}{h}-\left[\frac{t_{1}}{h}\right]\right] h\right)=0, t \geqslant 0,
$$

and

$$
\dot{v}(t)+p_{0}(t) v\left(\left[\frac{t}{h}\right] h\right)+\sum_{i=1}^{m} p_{i}(t) v\left(\left[\frac{t}{h}-\left[\frac{\tau_{i}}{h}\right]\right] h\right)-0, t \geqslant 0,
$$

and

$\dot{w}(t)+p_{0}\left(\left[\frac{t}{h}\right] h\right) w\left(\left[\frac{t}{h}\right] h\right)+\sum_{i=1}^{m} p_{i}\left(\left[\frac{t}{h}\right] h\right) w\left(\left[\frac{t}{h}-\left[\frac{\tau_{i}}{h}\right]\right] h\right)-0, t \geqslant 0$.

Note here, the theory of EPCA was initiated by Wiener, Cooke and Shah in [5] and [11] and some special EPCA with arguments $\left[\frac{t}{h}\right]$ h have been introduced in [6].

In the second section of this paper we show that the solutions of $u_{h}(t), v_{h}(t)$ and $w_{h}(t)$ of equations $(1.2)_{h},(1.3)_{h}$ and $(1.4)_{h}$, respectively, approximate the solutions $x(t)$ of Eq. (1.1) uniformly on any compact interval of $[0, \infty)$, as $h \rightarrow 0$. We also prove that these approximations are uniform on $[0, \infty)$, if (1.1) is an $L^{1}$ perturbation of $\dot{x}(t)=0$, that is for all $i-0,1, \ldots, m, \int_{0}^{\infty} p_{i}(t) \mid d t<\infty$. Our proofs are based on the well-known Gronwall - Bellman lemma.

In the third section we extend some of these results to delay differential equations of neutral type, by using a generalization of the Gronwall Belman lemma for the nonnegative solutions of the following inequality

$$
y(t) \leqslant f(t)+b y(t-\beta)+a \int_{0}^{t} y(s-a) d s, t \geqslant 0
$$

where $f(t)$ is a given nondecreasing continuous function and $a>0, b \geqslant 0, \beta \geqslant 0$ and $\alpha \geqslant 0$ are given constants.

2. DELAY DIFFERENTIAL EQUATIONS.

Consider the delay differential equation

$$
\dot{x}(t)+p_{0}(t) x(t)+\sum_{i=1}^{m} p_{i}(t) x\left(t-\tau_{1}\right)=0, t \geqslant 0
$$

where we state the following hypotheses

$\left(\mathrm{H}_{1}\right)$ for $i=1,2, \ldots, \mathrm{m}, \tau_{i}$ are positive real numbers and $\underset{1 \leqslant i \leqslant m}{\boldsymbol{m}}=\max \tau_{i} ;$

$\left(\mathrm{H}_{2}\right)$ for $\mathrm{i}=0,1, \ldots \mathrm{m}$, the functions $\mathrm{p}_{1}: \mathrm{R}_{+} \rightarrow \mathrm{R}$ are continuous.

The initial conditions associated with (2.1) are of the following type $x(s)-\phi(s),-\tau \leqslant s \leqslant 0 ; \phi \in C \equiv C([-\tau, 0], R)$. 
It is known ([3]) that under hypotheses $\left(\mathrm{H}_{1}\right)$ and $\left(\mathrm{H}_{2}\right)$ the initial value problem (2.1) and (2.2) has a unique solution on $[-\tau, \infty)$ which is continuous on $(-\tau, \infty)$ and continuously differentiable on $(0, \infty)$. The solution of (2.1) and (2.2) is denoted by $x(\phi)(t)$.

Let $k \geqslant 1$ be a fixed integer and set

$$
\mathrm{h}=\frac{\mathrm{\tau}}{\mathrm{k}} \text {. }
$$

By using $h$ we define the following three delay differential equations with piecewise constant arguments:

$$
\dot{u}(t)+p_{0}(t) u(t)+\sum_{i=1}^{m} p_{i}(t) u\left(\left[\frac{t}{h}-\left[\frac{\tau_{i}}{h}\right]\right] h\right)-0, t \geqslant 0
$$

and

$$
\dot{v}(t)+p_{0}(t) v\left(\left[\frac{t}{h}\right] h\right)+\sum_{i=1}^{m} p_{i}(t) v\left(\left[\frac{t}{h}-\left[\frac{\tau_{i}}{h}\right]\right] h\right)-0, t \geqslant 0
$$

and

$$
\dot{w}(t)+p_{0}\left(\left[\frac{t}{h}\right] h\right) w\left(\left[\frac{t}{h}\right] h\right)+\sum_{i=1}^{m} p_{i}\left(\left[\frac{t}{h}\right] h\right) w\left(\left[\frac{t}{h}-\left[\frac{r_{i}}{h}\right]\right] h\right)-0, t \geqslant 0 .
$$

With these equations we associate the following initial conditions

$$
u(j h)=\phi(j h) \text { for } j=-k, \ldots, 0
$$

and

$$
v(j h)=\phi(j h) \text { for } j=-k, \ldots, 0
$$

and

$$
w(j h)=\phi(j h) \text { for } j=-k, \ldots, 0,
$$

respectively, where $\phi \in C$.

By solution of $(2.4)_{h}$ and $(2.5)_{h}$ we mean a function $u_{h}(\phi)(t)$ which is defined on the set $(-k, \ldots, 0\} \cup(0, \infty)$ and satisfies the following properties:

(a) $u_{h}(\phi)(t)$ is continuous on $[0, \infty)$;

(b) the derivative $\dot{u}_{h}(\phi)(t)$ exists at each point $t \in[0, \infty)$ with the possible exception of the points $t=n h,(n \in N)$, where finite one-sided derivatives exist ;

(c) the function $u(t)=u_{h}(\phi)(t)$ satisfies Eq.(2.1) on each interval $[\mathrm{nh},(\mathrm{n}+1) \mathrm{h}]$ for $\mathrm{n} \in \mathrm{N}$.

The definitions of the solutions $v_{h}(\phi)(t)$ and $w_{h}(\phi)(t)$ of the initial value problems $(2.5)_{h}-(2.8)_{h}$ and $(2.6)_{h}-(2.9)_{h}$, respectively, are analogous and they are omitted.

First we prove the following existence and uniqueness result.

LEMMA 2.1. Assume that hypotheses $\left(\mathrm{H}_{1}\right)$ and $\left(\mathrm{H}_{2}\right)$ hold and $\mathrm{h}=\frac{\mathrm{\tau}}{\mathrm{k}},(\mathrm{k} \geq 1)$ and $\phi \in C$. Then each one of the initial value problems $(2.4)_{h}-(2.7)_{h},(2.5)_{h}-$ $(2.8)_{h}$ and $(2.6)_{h}-(2.9)_{h}$ has a unique solution.

PROOF. Consider the initial value problem $(2.4)_{h}-(2.7)_{h}$. For all $n \geqslant 0$ and $t \in[\mathrm{nh},(\mathrm{n}+1) h)$ one has

$$
\left[\frac{t}{h}-\left[\frac{\tau_{i}}{h}\right]\right] h=\left(n-k_{i}\right) h \text {, }
$$


where $k_{i}=\left[\frac{\tau_{i}}{h}\right], i=1, \ldots, n$.

Thus for $t \in[\mathrm{nh},(\mathrm{n}+1) \mathrm{h}),(2.4)_{\mathrm{h}}$ is equivalent to the following equation

$$
\dot{u}(t)+p_{0}(t) u(t)+\sum_{i=1}^{m} p_{i}(t) u\left(\left(n-k_{i}\right) h\right)=0 \text {. }
$$

By using the initial condition $(2.7)_{h}$ we find that $u(t)$ is a solution of $(2.4)_{h}$ and $(2.7)_{h}$ on $[0, h)$ if and only if $u(t)$ is a solution of

$$
\dot{u}(t)+p_{0}(t) u(t)+\sum_{i=1}^{m} p_{i}(t) \phi\left(-k_{i} h\right)-0,0 \leqslant t<h,
$$

and

But this initial value problem has exactly one solution which is continuously differentiable on $[0, h)$ and $u(h)$ and $\dot{u}(h)$ are defined, as $t \rightarrow h$. Then one can easily show, by the method of steps, that $u(t)$ exists and is unique on $[0, \infty)$.

By using an argument similar to that given above one can prove that each one of the initial value problems $(2.5)_{h}-(2.8)_{h}$ and $(2.6)_{h}-(2.9)_{h}$ has one and only one solution on $[0, \infty)$. The proof is complete.

Let $c_{0}^{1}$ be defined by $c_{0}^{1}-\left(\psi \in c^{1}: \dot{\psi}(0-)+p_{0}(0) \psi(0)+\sum_{i=1}^{m} p_{i}(0) \psi\left(-\tau_{i}\right)-0\right\}$ where $C^{1}$ denotes the set of continuously differentiable maps of $[-\tau, 0]$ into $R$. We now are ready to prove our convergence theorem.

THEOREM 2.1. Assume that hypotheses $\left(\mathrm{H}_{1}\right)$ and $\left(\mathrm{H}_{2}\right)$ hold and $\phi \in \mathrm{C}$ is given. Then the following statements are valid:

(a) the solutions $x(\phi)(t), u_{h}(\phi)(t), v_{h}(\phi)(t)$ and $w_{h}(\phi)(t)$ of the initial value problems $(2.1)-(2.2),(2.4)_{h}-(2.7)_{h},(2.5)_{h}-(2.8)_{h}$ and $(2.6)_{h}-(2.9)_{h}$, respectively, satisfy the following relations for all $\mathrm{T}>0$

$\lim _{h \rightarrow 0} \max _{0 \leqslant t \leqslant T}\left|x(\phi)(t)-u_{h}(\phi)(t)\right|-\lim _{h \rightarrow 0} \max _{0 \leqslant t \leqslant T}\left|x(\phi)(t)-v_{h}(\phi)(t)\right|-$

$$
\lim _{h \rightarrow 0} \max _{0 \leqslant t \leqslant T}\left|x(\phi)(t)-w_{h}(\phi)(t)\right|-0 .
$$

(b) If $\phi \in \mathrm{C}_{0}^{1}$ then for all $\mathrm{T}>0$ there exist constants $\mathrm{L}-\mathrm{L}(\mathrm{T}, \phi)$ and $M=M(T, \phi)$ such that

$$
\left|x(\phi)(t)-u_{h}(\phi)(t)\right| \leqslant L h, 0 \leqslant t \leqslant T, h>0,
$$

and

$$
\left|x(\phi)(t)-v_{h}(\phi)(t)\right| \leqslant M h, \quad 0 \leqslant t \leqslant T, h>0 ;
$$

(c) If for all $\mathrm{i}-0,1, \ldots, \mathrm{n}$ the functions $\mathrm{p}_{\mathrm{i}}(t)$ are Lipschitz-continuous on any compact subinterval of $[0, \infty)$ and $\phi \in C_{0}^{1}$, then there exists a constant $\mathrm{N}=\mathrm{N}(\mathrm{T}, \phi)$ such that

$$
\left|x(\phi)(t)-w_{h}(\phi)(t)\right| \leqslant N h, 0 \leqslant t \leqslant T, h>0 .
$$

REMARK 2.1. It is known from Lemma 2.1 in [7] that $c_{0}^{1}$ is a nonempty and dense set in $C$. 
PROOF OF THEOREM 2.1. (a) Consider the solutions $x(t)=x(\phi)(t)$ and $u_{h}(t)$ $=u_{h}(\phi)(t)$ of initial value problems $(2.1)-(2.2)$ and $(2.4)_{h}-(2.7)_{h}$, respectively. Then from (2.1) and $(2.4)_{h}$ we find

$$
\dot{x}(t)-\dot{u}_{h}(t)=-p_{0}(t)\left(x(t)-u_{h}(t)\right)-\sum_{i=1}^{m} p_{i}(t)\left(x\left(t-\tau_{i}\right)-u\left(\left[\frac{t}{h}-\left[\frac{\tau_{i}}{h}\right]\right) h\right)\right),
$$

for all $t \geqslant 0$.

Thus the function $\varepsilon(t)-\left|x(t)-u_{h}(t)\right|$ satisfies $\varepsilon(0)-0$ and for all $t \geqslant 0$, $\varepsilon(t) \leqslant \int_{0}^{t}\left(\left|p_{0}(s)\right| \varepsilon(s)+\sum_{i=1}^{m}\left|p_{i}(s) \| x\left(s-\tau_{i}\right)-u\left(\left[\frac{s}{h}-\left[\frac{\tau_{i}}{h}\right]\right] h\right)\right|\right) d s$

where

$$
\leqslant \int_{0}^{t}\left(\left|p_{0}(s)\right| \varepsilon(s)+\sum_{i=1}^{m}\left|p_{i}(s)\right| \varepsilon\left(\left[\frac{s}{h}-\left[\frac{\tau_{i}}{h}\right]\right] h\right) d s+f(t),\right.
$$

$$
f(t)=\int_{0}^{t} \sum_{i=1}^{m}\left|p_{i}(s) \| x\left(s-\tau_{i}\right)-x\left(\left[\frac{s}{h}-\left[\frac{\tau_{i}}{h}\right]\right] h\right)\right| d s, t \geqslant 0 .
$$

On the other hand,

$$
\left[\frac{s}{h}-\left[\frac{\tau_{i}}{h}\right]\right] h \leqslant s \text { for all } s \geqslant 0 \text {, }
$$

and by using the initial conditions one has

$$
\varepsilon\left(\left[\frac{s}{h}-\left[\frac{\tau_{i}}{h}\right]\right] h\right)=\left|\phi\left(\left[\frac{s}{h}-\left[\frac{\tau_{i}}{h}\right]\right] h\right)-u_{h}\left(\left[\frac{s}{h}-\left[\frac{\tau_{1}}{h}\right]\right] h\right)\right|-0,
$$

for all $i-1, \ldots, n$ and for all $s \geqslant 0$ such that

$$
\left[\frac{\mathrm{s}}{\mathrm{h}}-\left[\frac{\boldsymbol{\tau}_{\mathbf{i}}}{\mathrm{h}}\right] \mathrm{h}\right] \leqslant 0 \text {. }
$$

Therefore we find that the function $y(t) \underset{0 \leqslant s \leqslant t}{-\max _{0} \varepsilon(s)}$ satisfies the inequality

$$
y(t) \leqslant \int_{0}^{t} \sum_{i=1}^{m}\left|p_{i}(s)\right| y(s) d s+f(t), t \geqslant 0,
$$

where we used that $f(t)$ is a monotone increasing function. By Gronwall - Bellman inequality ([3]) we find

$$
y(t) \leqslant e^{\int_{0}^{t} \sum_{i=1}^{m}\left|p_{i}(s)\right| d s} f(t), t \geqslant 0 .
$$

By using the definition of the greatest integer function, we find for all $1-1,2, \ldots, \mathrm{m}$ and for all $s \geqslant 0$

$$
-h \leqslant s-\tau_{1}-\left[\frac{s}{h}-\left[\frac{\tau_{1}}{h}\right]\right] h \leqslant h .
$$

Set

$$
\omega(x ; t, h)-\max \left\{\left|x\left(t_{2}\right)-x\left(t_{1}\right)\right|:-\tau \leqslant t_{1}, t_{2} \leqslant t,\left|t_{2}-t_{1}\right| \leqslant h\right\} .
$$

Then from (2.15) it follows that

$$
\left|x\left(s-\tau_{i}\right)-x\left(\left[\frac{s}{h}-\left[\frac{\tau_{1}}{h}\right]\right] h\right)\right| \leqslant \omega(x ; t, h),
$$

for all $0 \leqslant s \leqslant t$ and for all $i-1, \ldots, m$. Also,

$$
f(t) \leqslant \int_{0}^{t} \sum_{i=0}^{m}\left|p_{i}(s)\right| d s \omega(x ; t, h), t \geqslant 0,
$$


and clearly (2.14) yields

$$
y(t) \leqslant e^{\int_{0}^{t} \sum_{i=0}^{m}\left|p_{i}(s)\right| d s} \int_{0}^{t} \sum_{i=1}^{m}\left|p_{i}(s)\right| d s \quad \omega(x ; t, h), t \geqslant 0 .
$$

Since $\varepsilon(t)=\left|x(t)-u_{h}(t)\right|=\left|x(\phi)(t)-u_{h}(\phi)(t)\right| \leqslant y(t)$, we obtain

$$
\left|x(\phi)(t)-u_{h}(\phi)(t)\right| \leqslant e^{\int_{0}^{t} \sum_{i=0}^{m}\left|p_{i}(s)\right| d s} \int_{0}^{t} \sum_{i=1}^{m}\left|p_{i}(s)\right| d s \quad \omega(x ; t, h)
$$

for all $h=\frac{T}{k}>0$ and $t \geqslant 0$.

But the solution $x(\phi)(t)$ of (2.1) - (2.2) is continuous on $[-\tau, \infty)$ and hence from (2.18) it can be easily seen that for all fixed $\mathrm{T}>0$

$\max _{0 \leqslant t \leqslant T}\left|x(\phi)(t)-u_{h}(\phi)(t)\right| \leqslant e^{\int_{0}^{T} \sum_{i=0}^{m}\left|p_{i}(s)\right| d s} \int_{0}^{T} \sum_{i=1}^{m}\left|p_{i}(s)\right| d s \omega(x ; T, h) \rightarrow 0$,

as $h_{\mathrm{t}} \rightarrow 0$.

By using an argument similar to that given above, we have

$\max _{0 \leqslant t \leqslant T} \mid x\left(\phi(t)-v_{h}(\phi)(t)\left|\leqslant e^{\int_{0}^{T} \sum_{i=0}^{m}\left|p_{i}(s)\right| d s} \int_{0}^{T} \sum_{i=0}^{m}\right| p_{i}(s) \mid d s \omega(x ; T, h) \rightarrow 0\right.$,

as $\mathrm{h} \rightarrow 0$.

It remains to consider the solutions $x(t)-x(\phi)(t)$ and $w(t)-w_{h}(\phi)(t)$ of the equations $(2.1)$ and $(2.6)_{h}$, respectively. In that case

$$
\begin{gathered}
\dot{x}(t)-\dot{w}(t)-p_{0}\left(\left[\frac{t}{h}\right] h\right)\left(x\left(\left[\frac{t}{h}\right] h\right)-w\left(\left[\frac{t}{h}\right] h\right)\right)+ \\
\sum_{i=1}^{m} p_{i}\left(\left[\frac{t}{h}\right] h\right)\left(x\left(\left[\frac{t}{h}-\left[\frac{\tau_{i}}{h}\right]\right] h\right)-w\left(\left[\frac{t}{h}-\left[\frac{\tau_{i}}{h}\right]\right] h\right)\right)+\left(p_{0}(t)-p_{0}\left(\left[\frac{t}{h}\right] h\right)\right) x\left(\left[\frac{t}{h}\right] h\right)+ \\
\sum_{i=1}^{m}\left(p_{i}(t)-p_{i}\left(\left[\frac{t}{h}\right] h\right)\right) x\left(\left[\frac{t}{h}-\left[\frac{\tau_{i}}{h}\right]\right] h\right)+p_{0}(t)\left(x(t)-x\left(\left[\frac{t}{h}\right] h\right)\right)+ \\
\sum_{i=1}^{m} p_{i}(t)\left(x\left(t-\tau_{i}\right)-x\left(\left[\frac{t}{h}-\left[\frac{\tau_{i}}{h}\right]\right] h\right)\right)
\end{gathered}
$$

for all $t \geqslant 0$.

Set $\varepsilon(t)=|x(t)-w(t)|, t \geqslant 0$. Then $\varepsilon(0)=0$ and by integrating we obtain

$\varepsilon(t) \leqslant \int_{0}^{t}\left(\left|\mathrm{P}_{0}\left(\left[\frac{s}{h}\right] h\right)\right| \varepsilon\left(\left[\frac{s}{h}\right] h\right)+\sum_{i=1}^{m}\left|p_{i}\left(\left[\frac{s}{h}\right] h\right)\right| \varepsilon\left(\left[\frac{s}{h}-\left[\frac{\tau}{h}\right]\right] h\right) d s+g(t), t \geqslant 0\right.$,

where

$g(t)-\int_{0}^{t}\left(\left|p_{0}(s)-p_{0}\left(\left[\frac{s}{h}\right] h\right)\right|\left|x\left(\left[\frac{s}{h}\right] h\right)+\sum_{i=1}^{m}\right| p_{i}(s)-p_{i}\left(\left[\frac{s}{h}\right] h\right) \| x\left(\left[\frac{s}{h}-\left[\frac{\tau_{i}}{h}\right]\right] h\right) \mid d s+\right.$ $\int_{0}^{t}\left(\left|p_{0}(s)\left\|x(s)-x\left(\left[\frac{s}{h}\right] h\right)+\sum_{i=1}^{m}\left|p_{i}(s) \| x\left(s-\tau_{i}\right)-x\left(\left[\frac{s}{h}-\left[\frac{\tau_{i}}{h}\right]\right] h\right)\right| d s, \quad t \geqslant 0\right.\right.\right.$. 
But for all $i=1, \ldots, m$ and for all $s \geqslant 0$ the inequality

yields

$$
\left[\frac{\mathrm{s}}{\mathrm{h}}-\left[\frac{\boldsymbol{\tau}_{\mathbf{i}}}{\mathrm{h}}\right]\right] \leqslant 0
$$

$$
\begin{aligned}
& \varepsilon \varepsilon\left(\left[\frac{s}{h}-\left[\frac{\tau_{i}}{h}\right]\right] h\right)-0 . \\
& \text { Set } y(t)=\max _{0 \leqslant s \leqslant t} \varepsilon(s) \text {, for } t \geqslant 0 \text {. Then } \\
& \varepsilon\left(\left[\frac{s}{h}-\left[\frac{\tau_{i}}{h}\right]\right] h\right) \leqslant y(s), 1 \leqslant i \leqslant m \text { and } s \geqslant 0 .
\end{aligned}
$$

Moreover, $g(t)$ is a monotone increasing function and hence (2.21) yields

$$
y(t) \leqslant \int_{0}^{t}\left(\sum_{i=0}^{m} \mid p_{i}\left(\left[\frac{s}{h}\right] h \mid\right) y(s) d s+g(t), t \geqslant 0 .\right.
$$

By Gronwa11-Bellman lemma (see, e.g. [3]) this yields

$$
y(t) \leqslant e^{\int_{0}^{t} \sum_{i=0}^{m}\left|p_{i}\left(\left[\frac{s}{h}\right] h\right)\right| d s} g(t) \text { for all } t \geqslant 0 .
$$

On the other hand,

$$
g(t) \leqslant \int_{0}^{t} \sum_{i=0}^{m}\left|\dot{p}_{i}(s)-p_{i}\left(\left[\frac{s}{h}\right] h\right)\right| d s \max _{-\tau \leqslant s \leqslant t}\left|x(s) H \int_{0}^{t} \sum_{i=0}^{m}\right| p_{i}(s) \mid d s \omega(x ; t, h)
$$

for all $t \geqslant 0$, where $\omega(x ; t, h)$ is defined in (2.16).

By using the definitions of $\varepsilon(t)$ and $y(t)$, from (2.22) and (2.23) we obtain for all $T>0$

$$
\begin{aligned}
& \max _{0 \leqslant t \leqslant T}\left|x(\phi)(t)-w_{h}(\phi)(t)\right| \leqslant e^{\int_{0}^{T} \sum_{i=0}^{m}\left|p_{i}\left(\left[\frac{s}{h}\right] h\right)\right| d s} \quad\left(\int_{0}^{T} \sum_{i=0}^{m}\left|p_{i}(s)-p_{i}\left(\left[\frac{s}{h}\right] h\right)\right| d s \max _{-\tau \leqslant s \leqslant T}|x(s)|\right. \\
& \left.\quad+\int_{0}^{T} \sum_{i=0}^{m}\left|p_{i}(s)\right| d s \omega(x ; T, h)\right) \rightarrow 0 \text {, as } h \rightarrow 0 .
\end{aligned}
$$

The proof of statement (a) is complete.

(b) Assume that $\phi \in C_{0}^{1}$. Then it can be easily seen that the solution $x(\phi)(t)$ of (2.1) $-(2.2)$ is continuously differentiable on $[-\tau, \infty)$ and clearly for all $T>0$ there exists a constant $K=K(T)$ such that

$$
\omega(\mathrm{x} ; \mathrm{T}, \mathrm{h}) \leqslant \mathrm{Kh}, \mathrm{h} \geqslant 0 \text {. }
$$

Set

and

$$
L=K e^{\int_{0}^{T}{ }_{i=0}^{m}\left|p_{i}(s)\right| d s} \int_{0}^{T} \sum_{i=1}^{m}\left|p_{i}(s)\right| d s
$$

$$
M=K e^{\int_{0}^{T} \sum_{i=0}^{m}\left|p_{i}(s)\right| d s} \int_{0}^{T} \sum_{i=0}^{m}\left|p_{i}(s)\right| d s .
$$

Then (2.11) and (2.12) follow from (2.19) and (2.20), respectively. Moreover, if we assume that $p_{i}(t)^{\prime}$ s are Lipschitz-continuous on any compact interval $[0, T]$, then 
there exists a constant $m_{1}=m_{1}(T)$ such that

$$
\sum_{i=0}^{m}\left|p_{i}(s)-p_{i}\left(\left[\frac{s}{h}\right] h\right)\right| \leqslant m_{1}\left(s-\left[\frac{s}{h}\right] h\right) \leqslant m_{1} h
$$

for all $s \in[0, T]$.

Set $m_{2}=\max _{0 \leqslant s \leqslant T} \sum_{i=0}^{m}\left|p_{i}(s)\right|$ and

$$
\mathrm{N}=\mathrm{e}^{\mathrm{m}_{2} \mathrm{~T}}\left(\mathrm{Tm}_{1}+\int_{0}^{\mathrm{T}} \sum_{\mathrm{i}=0}^{\mathrm{m}}\left|\mathrm{p}_{\mathrm{i}}(\mathrm{s})\right| \mathrm{ds}\right) .
$$

Then (2.24) and (2.25) yield inequality (2.13) and the proof of the theorem is complete.

In the next result we give a condition which guarantees that the first two approximations are uniform on the half line $[0, \infty)$.

THEOREM 2.2. Assume that hypotheses $\left(\mathrm{H}_{1}\right)$ and $\left(\mathrm{H}_{2}\right)$ are satisfied and for all i $-0,1, \ldots, \mathrm{m}$

$$
\int_{0}^{\infty} p_{i}(t) \mid d t<\infty
$$

Let $\phi \in \mathrm{C}$ be a given initial function. Then the solutions $x(\phi)(t), u_{h}(\phi)(t)$ and $v_{h}(\phi)(t)$ of equations $(2.1),(2.4)_{h}$ and $(2.5)_{h}$, respectively, satisfy the following relations

and

$$
\sup _{t \geqslant 0}\left|x(\phi)(t)-u_{h}(\phi)(t)\right| \rightarrow 0 \text {, as } h \rightarrow 0
$$

$$
\sup _{t \geqslant 0}\left|x(\phi)(t)-v_{h}(\phi)(t)\right| \rightarrow 0 \text {, as } h \rightarrow 0 \text {. }
$$

PROOF. By using (2.26) it can be easily seen that the solution of (2.1) (2.2) is bounded on $(0, \infty)$, and hence from (2.1) we find

$$
\int_{0}^{\infty} \dot{x}(t)\left|d t \leqslant \int_{0}^{\infty} \sum_{1=0}^{m}\right| p_{1}(t)\left|d t \sup _{-\tau \leqslant t \leqslant \infty}\right| x(t) \mid<\infty \text {. }
$$

Thus for all h $>0$

$$
a(h)-\sup _{T \geqslant h} \max _{h \leqslant t \leqslant T} \int_{t-h}^{t+h}|\dot{x}(s)| d s<\infty,
$$

and $a(h) \rightarrow 0$, as $h \rightarrow 0$.

Set $\omega(\phi ; h)-\max || \phi\left(t_{2}\right)-\phi\left(t_{1}\right)|:| t_{2}-t_{1} \mid \leqslant h$ and $\left.t_{1}, t_{2} \in[-\tau, 0]\right\}$. By using the definition (2.16) of $\omega(x ; T, h)$ and (2.29) we obtain

$$
\begin{aligned}
& \omega(x ; T, h) \leqslant \max \left\{\omega(\phi, h), \max _{h \leqslant t \leqslant T} \int_{t-h}^{t+h}|\dot{x}(u)| d u\right\} \leqslant \\
& \max \{\omega(\phi ; h), a(h)\} \equiv b(h), \text { for } a l l h>0 .
\end{aligned}
$$

In that case, by using (2.26), (2.19) and (2.20) yield

$$
\max _{\substack{0 \leqslant t \leqslant T \\ \text { and }}}\left|x(\phi)(t)-u_{h}(\phi)(t)\right| \leqslant e^{\int_{0}^{\infty} \sum_{i=0}^{m}\left|p_{i}(s)\right| d s} \int_{0_{i=1}^{\infty} \sum_{i=1}^{m}\left|p_{i}(s)\right| d s b(h), T \geqslant 0 \text {, }}
$$




$$
\max _{0 \leqslant t \leqslant T}\left|x(\phi)(t)-v_{h}(\phi)(t)\right| \leqslant e^{\int_{0}^{\infty} \sum_{i=0}^{m}\left|p_{i}(s)\right| d s} \int_{0}^{\infty} \sum_{i=0}^{m}\left|p_{i}(s)\right| d s b(h), T \geqslant 0,
$$

respectively. Since $\phi \in C$ and $a(h) \rightarrow 0$, as $h \rightarrow 0$, we find that $b(h) \rightarrow 0$, as $h \rightarrow 0$, and clearly (2.27) and (2.28) are satisfied. The proof of the theorem is complete.

The following result shows that equations $(2.4)_{h},(2.5)_{h}$ and $(2.6)_{h}$ are strongly related to some discrete difference equations. In this paper the first forward difference of a function $f(n)$ is denoted by

$$
\Delta f(n)-f(n+1)-f(n) \text {. }
$$

THEOREM 2.3. Assume that the hypotheses $\left(\mathrm{H}_{1}\right)$ and $\left(\mathrm{H}_{2}\right)$ are satisfied and $\mathrm{k} \geqslant$ 1 is an integer. Set

$$
\mathrm{h}=\frac{\mathrm{\tau}}{\mathrm{k}} \text { and } \mathrm{k}_{\mathrm{i}}=\left[\frac{\boldsymbol{\tau}_{\mathrm{i}}}{\mathrm{h}}\right] \text { for all } \mathrm{i}-1, \ldots, \mathrm{m}
$$

Then

(a) the solution $u_{h}(\phi)(t)$ of $(2.4)_{h}-(2.7)_{h}$ is given by $u_{h}(\phi)(t)=a(n) e^{-\int_{n h}^{t} p_{0}(s) d s}-e^{-\int_{0}^{t} p_{0}(s) d s} \sum_{i=1}^{m} \int_{n h}^{t} p_{i}(s) e^{\int_{0}^{s} p_{0}\left(s_{1}\right) d s_{1}} d s a\left(n-k_{i}\right)$

for all $\mathrm{nh} \leqslant \mathrm{t}<(\mathrm{n}+1) \mathrm{h}$ and $\mathrm{n} \geqslant 0$, where $\{\mathrm{a}(\mathrm{n})\}$ is a sequence which satisfies the difference equation

$$
\begin{aligned}
& a(n+1)-a(n) e^{-\iint_{n h}^{(n+1) h} p_{0}(s) d s}+ \\
& e^{-\int_{0}^{n h} P_{0}(s) d s} \sum_{i=1}^{m} \int_{n h}^{(n+1) h} f_{i}^{h}(s) e^{\int_{0}^{s} p_{0}\left(s_{1}\right) d s_{1}} d s \quad a\left(n-k_{i}\right)-0, n \geqslant 0 \\
& a(n)=\phi(n h) \quad, \quad n=-k, \ldots, 0 \\
& \text { (b) the solution } v_{h}(\phi)(t) \text { of }(2.5)_{h}-(2.8)_{h} \text { is given by }
\end{aligned}
$$

$$
v_{h}(\phi)(t)-\left(1-\int_{n h}^{t} p_{0}(u) d u\right) b(n)+\sum_{i=1}^{m} \int_{n h}^{t} p_{i}(u) d u b\left(n-k_{i}\right)
$$

for all $\mathrm{nh} \leqslant t<(n+1) h$ and $n \geqslant 0$, where $(b(n))$ is a sequence which satisfies the difference equation

$$
\begin{aligned}
& \left.\begin{array}{c}
\Delta b(n)+\int_{n h}^{(n+1){ }_{p_{0}}^{h}(u) d u b(n)+\sum_{i=1}^{m}} \int_{n h}^{(n+1) h} p_{i}(s) d s b\left(n-k_{i}\right)-0, n \geqslant 0 \\
b(n)=\phi(n h), \quad n--k, \ldots, 0
\end{array}\right\} \\
& \text { (c) the solution } w_{h}(\phi)(t) \text { of }(2.6)_{h}-(2.9)_{h} \text { is given by } \\
& w_{h}(\phi)(t)-c(n)-\left(p_{0}(n h) c(n)+\sum_{i=1}^{m} p_{i}(n h) c\left(n-k_{i}\right)\right)(t-n h)
\end{aligned}
$$




$$
\begin{aligned}
& \Delta c(n)+h p_{0}(n h) c(n)+h \sum_{i=1}^{m} p_{i}(n h) c\left(n-k_{i}\right)-0, n \geqslant 0 \\
& c(n)=\phi(n h), \quad n=-k, \ldots, 0 .
\end{aligned}
$$

PROOF. We prove (a) only. The proofs of (b) and (c) are similar and they will be omitted. Consider the solution $u(t)=u_{h}(\phi)(t)$ of $(2.4)_{h}-(2.7)_{h}$. Then we have $\frac{d}{d t}\left(u(t) e^{\int_{0}^{t} p_{0}(s) d s}\right)=-\sum_{i=1}^{m} p_{i}(t) u\left(\left[\frac{t}{h}-\left[\frac{\tau_{i}}{h}\right]\right] h\right) e^{\int_{0}^{t} p_{0}\left(s_{1}\right) d s_{1}}$

for all $t \geqslant 0$.

By integrating, from (2.37) it follows, for $t \in[n h,(n+1) h)$,

$$
\begin{aligned}
& u(t) e^{\int_{0}^{t} p_{0}(s) d s}=u(n h) e^{\int_{0}^{n h} p_{0}(s) d s}-\sum_{i=1}^{m} \int_{n h}^{t} p_{i}(s) e^{\int_{0}^{s} p_{0}\left(s_{1}\right) d s_{1}} u\left(\left[\frac{s}{h}-\left[\frac{\tau_{i}}{h}\right]\right] h\right) d s \\
& =a(n) e^{\int_{0}^{n h} p_{0}(s) d s}-\sum_{i=1}^{m} \int_{n h}^{t} p_{i}(s) e^{\int_{0}^{s} p_{0}\left(s_{1}\right) d s_{1}} d s a\left(n-k_{i}\right) \text {, for } n \geqslant 0 \text {, }
\end{aligned}
$$

where we use the notation

$$
a(n)=u(n h) \text { for } n=-k, \ldots, 0,1, \ldots \text {. }
$$

This means that $u(t)-u_{h}(\phi)(t)$ satisfies (2.31) with the sequence $\{a(n)\}$ defined by (2.38). We now show that $\{a(n)\}$ satisfies (2.32). But, by continuity of the solution, as $t \rightarrow(n+1) h,(2.31)$ yields (2.32). The proof of the theorem is complete.

REMARK 2.2. Consider the nonlinear delay differential equation

$$
\dot{x}(t)+f\left(t, x(t), x\left(t-\tau_{1}\right), \ldots, x\left(t-\tau_{m}\right)\right)=0, t \geqslant 0,
$$

with the initial condition

$$
x(t)=\phi(t),-\tau \leqslant t \leqslant 0 \text { and } \phi \in C \text {, }
$$

where $f: R_{+} \times R^{m} \rightarrow R$ is a continuous function and for all $i-1, \ldots, m, \tau_{1}>0$ and $\tau=\max _{1 \leqslant i \leqslant n} \tau_{i}$. Then one can generalize Theorem 2.1 for the initial value problem (2.39) - (2.40) provided that $f$ is a Lipschitz - continuous function and the approximating equations are given as follows:

$$
\dot{u}(t)+f\left(t, u(t), u\left(\left[\frac{t}{h}-\left[\frac{\tau_{1}}{h}\right]\right] h\right), \ldots, u\left(\left[\frac{t}{h}-\left[\frac{\tau_{m}}{h}\right]\right] h\right)\right)=0
$$

and

$$
\dot{v}(t)+f\left(t, v\left(\left[\frac{t}{h}\right] h\right), v\left(\left[\frac{t}{h}-\left[\frac{\tau_{1}}{h}\right]\right] h\right), \ldots, v\left(\left[\frac{t}{h}-\left[\frac{\tau_{m}}{h}\right]\right] h\right)\right)=0
$$
and

$$
\dot{w}(t)+f\left(\left[\frac{t}{h}\right] h, w\left(\left[\frac{t}{h}\right] h\right), w\left(\left[\frac{t}{h}-\left[\frac{\tau_{1}}{h}\right]\right] h\right), \ldots, w\left(\left[\frac{t}{h}-\left[\frac{\tau_{m}}{h}\right]\right] h\right)-0\right.
$$

where $h=\frac{\tau}{k}$ and $k \geqslant 1$ is a given integer. Note that the equations (2.42) and (2.43) can be rearranged to some difference equations while (2.41) cannot.

3. NEUTRAL CASE. 


$$
\frac{d}{d t}[x(t)+q x(t-r)]+p x(t-\gamma)=0, \quad t \geqslant 0
$$

where

$$
\mathrm{p}, \mathrm{q} \in \mathrm{R}, \mathrm{p} \neq 0 \text { and } \gamma, \mathrm{r} \in(0, \infty) \text { and } \tau=\max (\gamma, \mathrm{r}\} \text {. }
$$

The initial conditions associated with (3.1) are of the following type

$$
x(s)=\phi(s),-\tau \leqslant s \leqslant 0 ; \phi \in C \equiv C([-\tau, 0], R) \text {. }
$$

Then it is known ([3]) that the initial value problem (3.1) and (3.3) has one and only one solution on $[-\tau, \infty)$. The unique solution of (3.1) and (3.2) is denoted by $x(\phi)$.

Let $k \geqslant 1$ be a given integer and $h-\frac{\tau}{k}$. Set

$$
\mathrm{m}_{\mathrm{h}}=\left[\frac{\mathrm{r}}{\mathrm{h}}\right] \text { and } t_{\mathrm{h}}=\left[\begin{array}{l}
\boldsymbol{z} \\
\mathrm{h}
\end{array}\right] \text {. }
$$

In this section we approximate the solutions of (3.1) with the solutions of the equation

$$
\frac{d}{d t}\left(u(t)+q u\left(t-m_{h} h\right)\right)+p u\left(\left[\frac{t}{h}-t_{h}\right] h\right)=0 \text { for } t \geqslant 0 \text {. }
$$

Note that Eq. (3.4) has two delayed arguments $t-m_{h} h$ and $\left[\frac{t}{h}-t_{h}\right] h$. The first one is continuous while the second one is piecewise constant. Therefore the initial condition associated with (3.4) is of "mixed" type

$$
u(s)=\phi_{h}(s),-m_{h} h \leqslant s \leqslant 0 \text { and } u(j h)=\phi(j h), j--t_{h}, \ldots, 0
$$

where for all $i=-m_{h}, \ldots,-1$ and $s \in(i h,(i+1) h$ ]

$$
\phi_{h}(s)=\phi(i h) \frac{(i+1) h-s}{h}+\phi((i+1) h) \frac{s-i h}{h}
$$

A function $u(t)=u_{h}(\phi)(t)$ is a solution of (3.5) and (3.6) if $u(t)$ is defined on the set $\left[-m_{h} h, 0\right] \cup\left(-b_{h}, \ldots, 0\right) \cup(0, \infty)$ and satisfies the following properties:

(a) $u(t)$ is continuous on $[0, \infty)$;

(b) the derivative of $u(t)+q u\left(t-m_{h} h\right)$ exists at each point $t \in[0, \infty)$ with the possible exception of the points $t-n h,(n \in N)$, where finite one-sided derivatives exist.

(c) the function $u(t)$ satisfies Eq. (3.5) on each interval [nh, $(n+1) h$ ) for $n \geq 0$ and the initial condition (3.6) is satisfied.

With initial value problem (3.5) and (3.6) we associate the delay difference equation of neutral type

$$
\Delta\left(a(n)+q a\left(n-m_{h}\right)\right)+p h a\left(n-b_{h}\right)-0 \text { for } n \geqslant 0
$$

and the initial condition

$$
a(n)=\phi(n h) \text { for } n=-k, \ldots, 0 \text {, }
$$

where $\Delta$ denotes the first forward difference, i.e., for a function $f(n)$

$$
\Delta f(n)-f(n+1)-f(n) \text {. }
$$

Note here, the initial value problem (3.8) and (3.9) has a unique solution which is denoted by $a_{h}(\phi)(n)$.

We now are ready to state and to prove the following basic existence and uniqueness result for the initial value problem (3.5) and (3.6).

LEMMA 3.1. Assume that (3.2) is satisfied and $h=\frac{r}{k}$ where $k \geqslant 1$ is an integer. Let $\phi \in \mathrm{C}$ be given. Then the initial value problem (3.5) and (3.6) has a 
unique solution $\mathrm{u}(\phi)(t)$. Moreover, $\mathrm{u}(\phi)(t)$ is given by

$$
\mathrm{u}(\phi)(t)=\mathrm{a}(\phi)(\mathrm{n}) \frac{(\mathrm{n}+1) \mathrm{h}-\mathrm{t}}{\mathrm{h}}+\mathrm{a}(\phi)(\mathrm{n}+1) \frac{\mathrm{t}-\mathrm{nh}}{\mathrm{h}}
$$

for all $t \in[\mathrm{nh},(\mathrm{n}+1) \mathrm{h})$ and for $a 11 \mathrm{n} \geqslant 0$, where $\mathrm{a}(\phi)(\mathrm{n})$ is the unique solution of (3.8) and (3.9).

PROOF. It can be easily seen that a function $u(t)$ is a solution of (3.5) if and only if for all $n \in N$ and $t \in[\mathrm{nh},(\mathrm{n}+1) \mathrm{h})$

$$
\frac{d}{d t}\left(u(t)+q u\left(t-m_{h} h\right)\right)+p u\left(\left(n-t_{h}\right) h\right)=0
$$

or equivalently

$$
u(t)=-q u\left(t-m_{h} h\right)+u(n h)+q u\left(\left(n-m_{h}\right) h\right)-p h u\left(\left(n-t_{h}\right) h\right) .
$$

By the method of steps it can be easily seen that Eq. (3.12) has one and only one solution satisfying (3.6), and clearly the initial value problem (3.5) and (3.6) has a unique solution $u(t)=u_{h}(\phi)(t)$. By continuity of the solution, as $t \rightarrow(n+1) h$, (3.12) yields

$$
\Delta\left(u((n+1) h)+q u\left(\left(n-m_{h}\right) h\right)\right)+p h u\left(\left(n-t_{h}\right) h\right)=0 \text { for } n \geqslant 0,
$$

and $u(n h)=\phi(n h)$, for $n=-k, \ldots, 0$. This means that $a(n)=u(n h)$ where $a(n)$ is the unique solution of (3.8) and (3.9). But from (3.11) it follows that the function $u(t)+q u\left(t-m_{h} h\right)$ is a piecewise linear function on $0 \leqslant t<\infty$. On the other hand, $u(t)$ is a piecewise linear function on $-m_{h} h \leqslant s \leqslant 0$. Therefore $u(t)$ is a piecewise linear function on $[0, \infty)$ and clearly (3.10) is satisfied. The proof is complete.

We now are in a position to state the following convergence theorem.

THEOREM 3.1. Assume that (3.2) is satisfied and $\phi \in \mathrm{C}$ is a fixed initial function. Then the solutions $x(\phi)(t)$ and $u_{h}(\phi)(t)$ of the initial value problems (3.1) - (3.3) and (3.5) - (3.6), respectively, satisfy the following limit relation

$$
\sup _{0 \leqslant t \leqslant T}\left|x(\phi)(t)-u_{h}(\phi)(t)\right| \rightarrow 0 \text {, for all } T>0 \text {, }
$$

as $\mathrm{h}=\frac{\mathrm{\tau}}{\mathrm{k}} \rightarrow 0$, or equivalently as $\mathrm{k} \rightarrow+\infty$.

The proof of the above theorem uses a generalization of the Gronwall-Bellman lemma for a continuous solution $y:[-\sigma, \infty) \rightarrow[0, \infty)$ of the inequality

$$
y(t) \leqslant f(t)+b y(t-\beta)+a \int_{0}^{t} y(s-\alpha) d s, t \geqslant 0,
$$

provided that

(i) $a>0, b \geqslant 0, \alpha \geqslant 0$ and $\beta>0$ are constants and $\sigma=\max \{\alpha, \beta\}$.

(ii) $f:[0, \infty) \rightarrow(0, \infty)$ is a continuous and nondecreasing function.

LEMMA 3.2. Assume that (i) and (ii) are satisfied and the continuous function $\mathrm{y}:[-\sigma, \infty) \rightarrow[0, \infty)$ satisfies (3.14). Then there exists a unique constant $\mathrm{c}>0$ such that

$$
c b e^{-c \beta}+a e^{-c \alpha}=c
$$

and

$$
y(t) \leqslant d(t) e^{c t} \text { for } t \geqslant 0
$$

where 


$$
d(t)=\max \left\{\frac{f(t)}{1-b e^{-c \beta}}, \max _{-\sigma \leqslant s \leqslant 0} e^{-c s} y(s)\right\} \text { for } t \geqslant 0
$$

PROOF. It is clear that equation (3.15) has a unique solution $c>0$ provided that (i) holds. Moreover, $1-b e^{-c \beta}>0$. Let $\varepsilon>0$ be given and set

$$
d_{\varepsilon}(t)=\max \left(\frac{\tilde{f}(t)+\varepsilon}{1-b e^{-c \beta}}, \max _{-\sigma \leqslant s \leqslant 0} e^{-c s} y(s)+\varepsilon\right) \text { for } t \geqslant-\sigma,
$$

where



Then it can be easily seen that

$$
y(t)<d_{\varepsilon}(t) e^{c t}
$$

for all $t \in[-\sigma, 0]$ and from (3.14) it follows

$$
y(t)<(\tilde{f}(t)+\varepsilon)+b y(t-\beta)+a \int_{0}^{t} y(s-\alpha) d s, \quad t \geqslant 0 \text {. }
$$

We now show that (3.19) is valid for all $t>0$. Otherwise, there exists $t_{1}>0$ such that

$$
y(t)<d_{\varepsilon}(t) e^{c t},-\sigma \leqslant t<t_{1} \text {, and } y\left(t_{1}\right)-d_{\varepsilon}\left(t_{1}\right) e^{c t_{1}} .
$$

But in that case $(3.20)$ yields

$$
y\left(t_{1}\right)<\left(\tilde{f}\left(t_{1}\right)+\varepsilon\right)+d_{\varepsilon}\left(t_{1}-\beta\right) e^{c\left(t_{1}-\beta\right)}+a \int_{0}^{t_{1}} d_{\varepsilon}(s-\alpha) e^{c(s-\alpha)} d s
$$

Since $f(t)$ is monotone nondecreasing we have that $\tilde{\mathrm{f}}(t)$ and $d_{\varepsilon}(t)$ are monotone nondecreasing. Hence by applying (3.15) we obtain

$$
\begin{aligned}
& y\left(t_{1}\right)<\tilde{f}\left(t_{1}\right)+\varepsilon+d_{\varepsilon}\left(t_{1}\right) e^{-c \beta} e^{c t_{1}}+\frac{a}{c} e^{-c \alpha} d_{\varepsilon}\left(t_{1}\right)\left(e^{c t_{1}}-1\right) \\
&=\tilde{f}\left(t_{1}\right)+\varepsilon-\frac{a}{c} e^{-c \beta} d_{\varepsilon}\left(t_{1}\right)+d_{\varepsilon}\left(t_{1}\right) e^{c t_{1}}\left(e^{-c \beta}+\frac{a}{c} e^{-c \alpha}-1\right)+d_{\varepsilon}\left(t_{1}\right) e^{c t_{1}} \\
&-\tilde{f}\left(t_{1}\right)+\varepsilon-\left(1-b e^{-c \beta}\right) d_{\varepsilon}\left(t_{1}\right)+d_{\varepsilon}\left(t_{1}\right) e^{c t_{1}} .
\end{aligned}
$$

But from (3.18) it follows

and hence

$$
\tilde{f}\left(t_{1}\right)+\varepsilon \leqslant\left(1-b e^{-c \beta}\right) d_{\varepsilon}\left(t_{1}\right) \text {, }
$$

$$
y\left(t_{1}\right)<d_{\varepsilon}\left(t_{1}\right) e^{c t_{1}} .
$$

This contradicts (3.21), and clearly (3.19) is satisfied for all $t \geqslant 0$. From (3.18) it can be easily seen that

$$
d(t)=\lim _{\varepsilon \rightarrow 0+} d_{\varepsilon}(t), \quad t \geqslant 0,
$$

therefore (3.19) implies (3.16), as $\varepsilon \rightarrow 0+$. The proof is complete.

REMARK 3.1. If $f(t) \equiv c_{0}$ is constant on $[0, \infty)$ and $b=0$ and $\alpha=0$ then $c=a$ is the unique positive solution of (3.15) and $d(t)-c_{0}$ for all $t \geqslant 0$. Thus Lemma 3.2 reduces to the well known Gronwall-Bellman inequality ([3]).

REMARK 3.2. One can easily generalize Lemma 3.2 for the case of several delays 
in the following form:

Assume that $y:[-\sigma, \infty) \rightarrow[0, \infty)$ is a continuous function such that

$$
y(t) \leqslant f(t)+\sum_{i=1}^{l} b_{i} y\left(t-\beta_{i}\right)+\int_{0}^{t}\left(a_{0} y(s)+\sum_{j=1}^{n} a_{j} y\left(s-\alpha_{j}\right)\right) d s, t \geqslant 0,
$$

provided that

(i) $b_{i} \geqslant 0, \beta_{i}>0,(1 \leqslant i \leqslant 1)$, and $a_{j} \geqslant 0,(0 \leqslant j \leqslant n)$ and $\alpha_{j} \geqslant 0,(1 \leqslant j \leqslant n)$, are constants $\sum_{j=0}^{n} a_{j}>0$ and $\sigma=\max \left(\max _{1 \leqslant 1 \leqslant i} \beta_{i}, \max _{1 \leqslant j \leqslant n} \alpha_{j}\right\} ;$

(ii)' $f:(0, \infty) \rightarrow[0, \infty)$ is a continuous and nondecreasing function.

Then the equation

$$
c \sum_{i=1}^{l} b_{i} e^{-c \beta_{i}}+a_{0}+\sum_{j=1}^{n} a_{j} e^{-c \alpha_{j}}-c
$$

has exactly one positive root $c$ and

$$
y(t) \leqslant d(t) e^{c t}, \quad t \geqslant 0
$$

where

$$
d(t)=\max \left(\left(1-\sum_{i=1}^{b} b_{i} e^{-c \beta_{i}}\right)^{-1} f(t), \max _{-\sigma \leqslant s \leqslant 0} e^{-c s_{y}(s)}\right\}
$$

for $t \geqslant 0$.

We now are in a position to prove Theorem 3.1 .

PROOF OF THEOREM 3.1. Consider the solutions $x(t)-x(\phi)(t)$ and $u_{h}(t)=$ $u_{h}(\phi)(t)$ of the initial value problems (3.1) - (3.3) and (3.5) - (3.6), respectively. Set

$$
y_{h}(t)= \begin{cases}x(t)-u_{h}(t), & t \geqslant 0 \\ 0, & -\tau \leqslant t<0 .\end{cases}
$$

Since $x(0)=u_{h}(0)$, we find that $y_{h}(t)$ is continuous on $[-\tau, \infty)$. On the other hand, from (3.1) and (3.5) it follows

$$
\frac{d}{d t}\left(x(t)-u_{h}(t)+q x(t-r)-q u_{h}\left(t-m_{h} h\right)\right)+p x(t-\gamma)-p u\left(\left[\frac{t}{h}-b_{h}\right] h\right)-0
$$

and clearly

$$
\begin{gathered}
\frac{d}{d t}\left(y_{h}(t)+q y_{h}\left(t-m_{h} h\right)+q\left(x(t-r)-x\left(t-m_{h} h\right)\right)\right)+p y_{h}\left(\left[\frac{t}{h}-t_{h}\right] h\right)+ \\
p\left(x(t-\gamma)-x\left(\left[\frac{t}{h}-t_{h}\right] h\right)\right)-0, t \geqslant 0 .
\end{gathered}
$$

Thus, from (3.3) and (3.6), by integration we find

$$
\begin{aligned}
y_{h}(t)+q y_{h}\left(t-m_{h} h\right)+q\left(x(t-r)-x\left(t-m_{h} h\right)\right)+p \int_{0}^{t} y_{h}\left(\left[\frac{s}{h}-b_{h}\right] h\right) d s \\
+p \int_{0}^{t}\left(x(s-\gamma)-x\left(\left[\frac{s}{h}-t_{h}\right] h\right)\right) d s-0, t \geqslant 0 .
\end{aligned}
$$

Set

$f_{h}(t)-|q| \max _{0 \leqslant s \leqslant t}\left|x(s-r)-x\left(s-m_{h} h\right)\right|+p \int_{0}^{t}\left|x(s-r)-x\left(\left[\frac{s}{h}-b_{h}\right] h\right)\right| d s, t \geqslant 0$.

In that case

$$
\left|y_{h}(t)\right| \leqslant f_{h}(t)+\left|q \| y_{h}\left(t-m_{h} h\right)\right|+|p| \int \frac{y_{0}}{y_{h}}\left(\left[\frac{s}{h}-t_{h}\right] h\right) d s, \quad t \geqslant 0 .
$$


Let $z_{h}(t)=\max _{-\tau \leqslant s \leqslant t}\left|y_{h}(s)\right|$. Since $y_{h}(t)=0,-m_{h} h \leqslant t \leqslant 0$, and $y_{h}\left(\left[\frac{s}{h}-b_{h}\right] h\right)-0$ wherever $\left[\frac{s}{h}-b_{h}\right] \leqslant 0$, we find

$$
z_{h}(t) \leqslant f_{h}(t)+|q| z_{h}\left(t-m_{h} h\right)+|p| \int_{0}^{t} z_{h}(s) d s, \quad t \geqslant 0 .
$$

By virtue of Lemma 3.2 , we have

$$
z_{h}(t) \leqslant d_{h}(t) e^{c_{h} t}, \quad t \geqslant 0
$$

where $c_{h}$ is the unique positive solution of

and

$$
c|q| e^{-c m_{h} h}+|p|=c
$$

Thus

$$
d_{h}(t)=\max \left\{\left(1-|q| e^{-c_{h} m_{h} h}\right)^{-1} f_{h}(t), \max _{-\sigma \leqslant s \leqslant 0} e^{-c_{h} s} z_{h}(s)\right\}-
$$

$$
\left(1-|q| e^{-c h^{m_{h}} h^{-1}} f_{h}(t) \quad, \quad t \geqslant 0 .\right.
$$

$$
\left|y_{h}(t)\right| \leqslant z_{h}(t) \leqslant\left(1-|q| e^{-c_{h} m_{h} h}\right)^{-1} f_{h}(t) \text { for all } t \geqslant 0 \text {. }
$$

Now, by using the continuity of $x(t)$ and an argument similar to that given in the proof of Theorem 2.1 one can see that for all $\mathrm{T}>0$

$$
\max _{0 \leqslant t \leqslant T} f_{h}(t) \rightarrow 0 \text {, as } h \rightarrow 0 \text {. }
$$

On the other hand,

$$
\mathrm{m}_{\mathrm{h}} \mathrm{h}-\left[\frac{\mathrm{r}}{\mathrm{h}}\right] \mathrm{h} \rightarrow \mathrm{r} \text {, as } \mathrm{h} \rightarrow 0 \text {, }
$$

therefore from (3.23) it can be seen that $c_{h} \rightarrow c_{0}$, as $h \rightarrow 0+$, where $c_{0}$ is the unique positive solution of

$$
\mathrm{cl} q \mathrm{e}^{-\mathrm{cr}}+\mathrm{a}=\mathrm{c} \text {. }
$$

Thus (3.24) and (3.25) yield

$$
\max _{0 \leqslant t \leqslant T}\left|y_{h}(t)\right|=\max _{0 \leqslant t \leqslant T}\left|x(\phi)(t)-u_{h}(\phi)(t)\right| \rightarrow 0 \text {, as } h \rightarrow 0 \text {, }
$$

for all $T>0$. The proof of the theorem is complete.

REMARK 3.3. Applying Remark 3.2 one can easily generalize Theorem 3.1 for the case of several delays.

\section{REFERENCES}

1. A.R. Aftabizadeh, J.Wiener and J.M.Xu, Oscillatory and periodic properties of delay differential equations with piecewise constant argument, Proc. Amer. Math. Soc. 99 (1987), 673-679.

2. A.R. Aftabizadeh and J.Wiener, Oscillatory and periodic solutions for systems of two first order linear differential equations with piecewise constant arguments, Applicable Anal. 26 (1988), 327-333.

3. R.Bellman and K.L.Cooke, Differential-Difference Equations, Academic Press, New York, 1963.

4. K.L.Cooke and J.Wiener, Neutral differential equations with piecewise constant argument, Boll. U.M.I. 1-B (1987), 321-346.

5. K.L.Cooke and J.Wiener, Retarded differential equations with piecewise constant delays, J. Math. Anal. Appl., 99 (1984), 265-297. 
6. K.L.Cooke and J.Wiener, Stability regions for linear equations with piecewise continuous delay, Computers \& Mathematics with Applications, 12A (1986), 695-701.

7. I. Gyori, Two approximation techniques for functional differential equations, Comput. Math. Applic. 16 (1988), 195-214.

8. I.Györi and G.Ladas, Linearized oscillations for equations with piecewise constant arguments, Differential and Integral Equations 1 (1988), 281-286.

9. J.Hale, Theory of Functional Differential Equations, Applied Mathematical Sciences 3, Springer-Verlag, New York, 1977.

10. J.P.LaSalle, The Stability and Control of Discrete Processes, SpringerVerlag, New York, 1986.

11. S.M.Shah and J.Wiener, Advanced differential equations with piecewise constant argument deviations, Internat. J. Math. \& Math. Sci., 6 (1983), 671-703. 


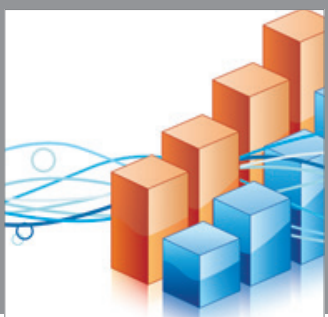

Advances in

Operations Research

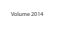

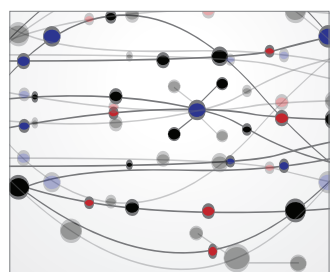

\section{The Scientific} World Journal
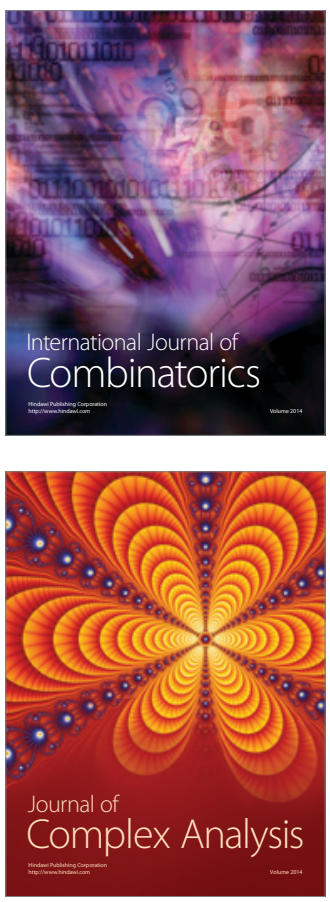

International Journal of

Mathematics and

Mathematical

Sciences
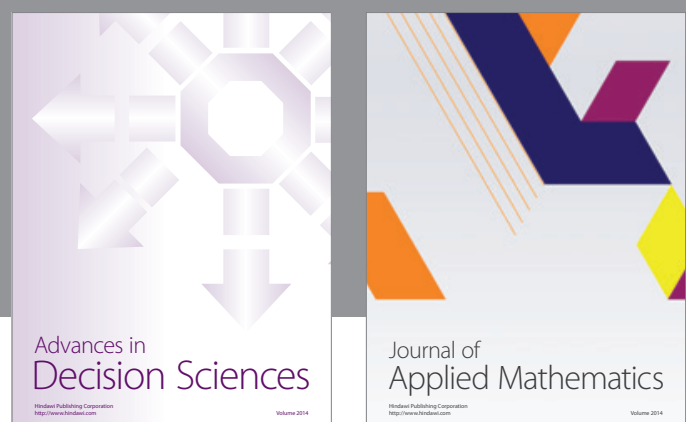

Journal of

Applied Mathematics


Submit your manuscripts at http://www.hindawi.com
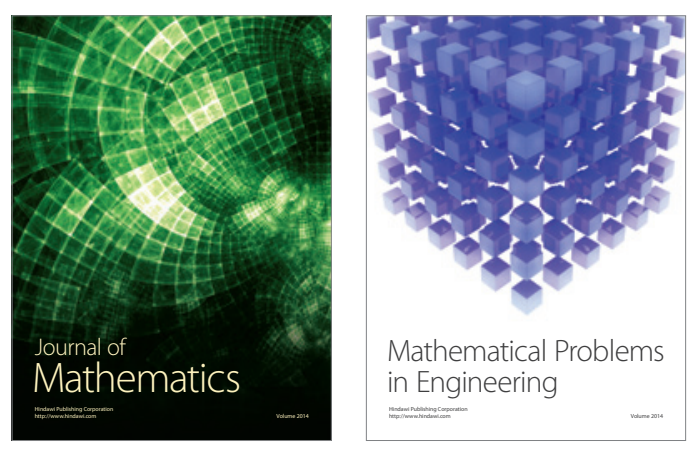

Mathematical Problems in Engineering
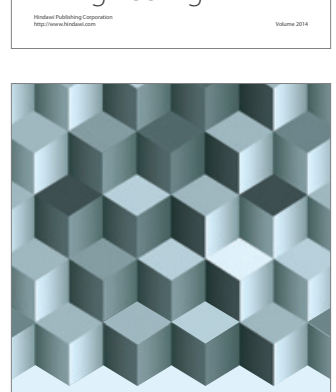

Journal of

Function Spaces
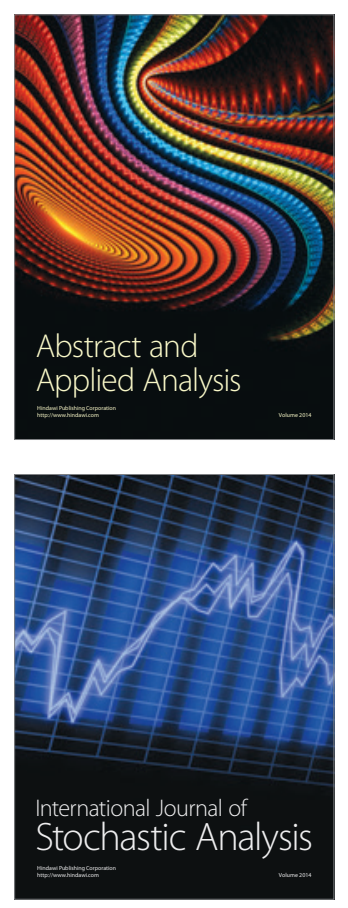



ournal of

Probability and Statistics

Promensencen
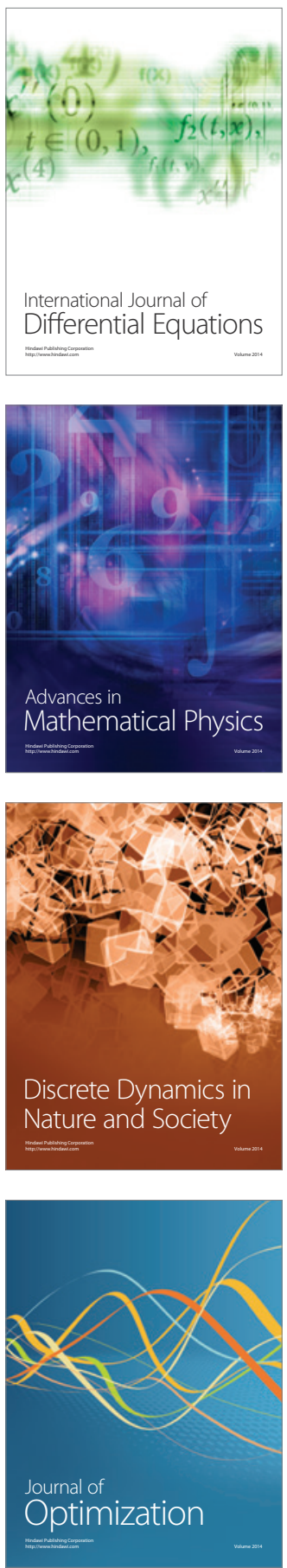